\title{
Research Article \\ On the Rate of Convergence of Kirk-Type Iterative Schemes
}

\author{
Nawab Hussain, ${ }^{1}$ Renu Chugh,${ }^{2}$ Vivek Kumar, ${ }^{2}$ and Arif Rafiq ${ }^{3}$ \\ ${ }^{1}$ Department of Mathematics, King Abdulaziz University, P.O. Box 80203, Jeddah 21589, Saudi Arabia \\ ${ }^{2}$ Department of Mathematics, M. D. University, Rohtak 124001, India \\ ${ }^{3}$ Hajuery University, 43-52 Industrial Area, Gulberg-III, Lahore, Pakistan
}

Correspondence should be addressed to Nawab Hussain, nhusain@kau.edu.sa

Received 29 January 2012; Revised 21 April 2012; Accepted 14 May 2012

Academic Editor: B. V. Rathish Kumar

Copyright $\odot 2012$ Nawab Hussain et al. This is an open access article distributed under the Creative Commons Attribution License, which permits unrestricted use, distribution, and reproduction in any medium, provided the original work is properly cited.

The purpose of this paper is to introduce Kirk-type new iterative schemes called Kirk-SP and Kirk-CR schemes and to study the convergence of these iterative schemes by employing certain quasi-contractive operators. By taking an example, we will compare Kirk-SP, Kirk-CR, Kirk-Mann, Kirk-Ishikawa, and Kirk-Noor iterative schemes for aforementioned class of operators. Also, using computer programs in $\mathrm{C}++$, we compare the above-mentioned iterative schemes through examples of increasing, decreasing, sublinear, superlinear, and oscillatory functions.

\section{Introduction and Preliminaries}

There is a close relationship between the problem of solving a nonlinear equation and that of approximating fixed points of a corresponding contractive-type operator. Consequently, there is a theoretical and practical interest in approximating fixed points of various contractive-type operators. Let $(X, d)$ be a complete metric space and $T: X \rightarrow X$ a selfmap of $X$. Suppose that $F(T)=\{p \in X, T p=p\}$ is the set of fixed points of $T$. There are several iterative schemes in the literature for which the fixed points of operators have been approximated over the years by various authors. In a complete metric space, the Picard iterative scheme is defined by

$$
x_{n+1}=T x_{n}, \quad n=0,1, \ldots,
$$


which is used to approximate the fixed points of mappings satisfying the inequality:

$$
d(T x, T y) \leq \alpha d(x, y)
$$

for all $x, y \in X$ and $\alpha \in[0,1)$. Condition (1.2) is called Banach's contraction condition.

The following iteration schemes are now well known:

$$
u_{n+1}=\left(1-\alpha_{n}\right) u_{n}+\alpha_{n} T u_{n}
$$

where $\left\{\alpha_{n}\right\}$ is a sequences of positive numbers in $[0,1]$, due to Mann [1].

$$
x_{n+1}=\sum_{i=0}^{k} \alpha_{i} T^{i} x_{n}, \quad \sum_{i=0}^{k} \alpha_{i}=1
$$

where $\left\{\alpha_{n}\right\}$ is a sequences of positive numbers in $[0,1]$, due to Kirk [2].

$$
\begin{gathered}
x_{n+1}=\left(1-\alpha_{n}\right) x_{n}+\alpha_{n} T y_{n}, \\
y_{n}=\left(1-\beta_{n}\right) x_{n}+\beta_{n} T x_{n},
\end{gathered}
$$

where $\left\{\alpha_{n}\right\}$ and $\left\{\beta_{n}\right\}$ are sequences of positive numbers in $[0,1]$, due to Ishikawa [3].

$$
\begin{gathered}
x_{n+1}=\left(1-\alpha_{n}\right) x_{n}+\alpha_{n} T y_{n}, \\
y_{n}=\left(1-\beta_{n}\right) x_{n}+\beta_{n} T z_{n}, \\
z_{n}=\left(1-\gamma_{n}\right) x_{n}+\gamma_{n} T x_{n},
\end{gathered}
$$

where $\left\{\alpha_{n}\right\},\left\{\beta_{n}\right\}$, and $\left\{\gamma_{n}\right\}$ are sequences of positive numbers in [0,1], due to Noor [4].

In [5], Olatinwo introduced the Kirk-Mann and Kirk-Ishikawa iterative schemes as follows:

$$
\begin{gathered}
x_{n+1}=\alpha_{n, 0} x_{n}+\sum_{i=1}^{k} \alpha_{n, i} T^{i} y_{n}, \quad \sum_{i=0}^{k} \alpha_{n, i}=1, \\
y_{n}=\sum_{j=0}^{s} \beta_{n, j} T^{j} x_{n}, \quad \sum_{j=0}^{s} \beta_{n, j}=1, \quad n=0,1,2, \ldots,
\end{gathered}
$$

where $k \geq s, \alpha_{n, i} \geq 0, \alpha_{n, 0} \neq 0, \beta_{n, j} \geq 0, \beta_{n, 0} \neq 0, \alpha_{n, i}, \beta_{n, j} \in[0,1]$, and $k, s$ are fixed integers, called as Kirk-Ishikawa iteration scheme:

$$
u_{n+1}=\sum_{i=0}^{k} \alpha_{n, i} T^{i} u_{n}, \quad \sum_{i=0}^{k} \alpha_{n, i}=1, \quad n=0,1,2, \ldots
$$


where $\alpha_{n, i} \geq 0, \alpha_{n, 0} \neq 0, \alpha_{n, i} \in[0,1]$, and $k$ is a fixed integer and is called as Kirk-Mann iteration scheme.

However, from [6], the Kirk-Noor iterative scheme is given by

$$
\begin{array}{cc}
x_{n+1}=\alpha_{n, 0} x_{n}+\sum_{i=1}^{k} \alpha_{n, i} T^{i} y_{n}, & \sum_{i=0}^{k} \alpha_{n, i}=1, \\
y_{n}=\beta_{n, 0} x_{n}+\sum_{r=1}^{s} \beta_{n, r} T^{r} z_{n}, & \sum_{j=0}^{s} \beta_{n, j}=1, \\
z_{n}=\sum_{l=0}^{t} \gamma_{n, l} T^{l} x_{n}, \quad \sum_{l=0}^{t} \gamma_{n, l}=1, & n=0,1,2, \ldots
\end{array}
$$

In [7], Phuengrattana and Suantai defined the SP iteration scheme as follows:

$$
\begin{gathered}
x_{n+1}=\left(1-\alpha_{n}\right) y_{n}+\alpha_{n} T y_{n}, \\
y_{n}=\left(1-\beta_{n}\right) z_{n}+\beta_{n} T z_{n}, \\
z_{n}=\left(1-\gamma_{n}\right) x_{n}+\gamma_{n} T x_{n},
\end{gathered}
$$

where $\left\{\alpha_{n}\right\},\left\{\beta_{n}\right\}$, and $\left\{\gamma_{n}\right\}$ are sequences of positive numbers in $[0,1]$.

Recently, Chugh and Kumar introduced the following iteration scheme [8]:

$$
\begin{gathered}
x_{n+1}=\left(1-\alpha_{n}\right) y_{n}+\alpha_{n} T y_{n}, \\
y_{n}=\left(1-\beta_{n}\right) T x_{n}+\beta_{n} T z_{n}, \\
z_{n}=\left(1-\gamma_{n}\right) x_{n}+\gamma_{n} T x_{n},
\end{gathered}
$$

where $\left\{\alpha_{n}\right\},\left\{\beta_{n}\right\}$, and $\left\{\gamma_{n}\right\}$ are sequences of positive numbers in $[0,1]$.

Remarks 1. (1) If $\gamma_{n}=0$, then (1.6) reduces to the Ishikawa iteration scheme (1.5).

(2) If $\beta_{n}=\gamma_{n}=0$, then (1.6) reduces to the Mann iteration scheme (1.3).

(3) If $\beta_{n}=0$, then (1.5) reduces to the Mann iteration scheme (1.3).

(4) If $\beta_{n}=\gamma_{n}=0$, then (1.10) reduces to the Mann iteration scheme (1.3).

In [9], Zamfirescu obtained the following interesting fixed point theorem.

Theorem 1.1. Let $(X, d)$ be a complete metric space and $T: X \rightarrow X$ a mapping for which there exists real numbers $a, b$, and $c$ satisfying $a \in(0,1), b, c \in(0,1 / 2)$ such that for each pair $x, y \in X$ at least one of the following conditions holds:

(i) $d(T x, T y) \leq a d(x, y)$,

(ii) $d(T x, T y) \leq b[d(x, T x)+d(y, T y)]$,

(iii) $d(T x, T y) \leq c[d(x, T y)+d(y, T x)]$. 
Then, $T$ has a unique fixed point $p$ and the Picard iteration scheme $\left\{x_{n}\right\}$ defined by (1.1) converges to $p$ for any arbitrary but fixed $x_{0} \in X$.

The operators satisfying the condition (1.12) are called Zamfirescu operators.

Berinde in [10] introduced a new class of operators on an arbitrary Banach space and satisfying

$$
d(T x, T y) \leq 2 \delta d(x, T x)+\delta d(x, y)
$$

for all $x, y \in X$ and $\delta \in[0,1)$. He proved that this class is wider than the class of Zamfirescu operators and used the Ishikawa iteration scheme to approximate fixed points of this class of operators in an arbitrary Banach space given in the form of following theorem.

Theorem 1.2 (see [10]). Let $K$ be a nonempty closed convex subset of an arbitrary Banach space $X$ and $T: K \rightarrow K$ a mapping satisfying (1.13). Let $\left\{x_{n}\right\}_{n=0}^{\infty}$ be defined through the Ishikawa iteration scheme (1.5) and $x_{0} \in K$, where $\left\{\alpha_{n}\right\},\left\{\beta_{n}\right\}$ are sequences of positive real numbers in $[0,1]$ with $\left\{\alpha_{n}\right\}$ satisfying $\sum_{n=0}^{\infty} \alpha_{n}=\infty$. Then, $\left\{x_{n}\right\}_{n=0}^{\infty}$ converges strongly to the fixed point of $T$.

However, in [11], Rafiq studied the convergence of the Noor iteration scheme [4] involving quasi-contractive operators.

Also several authors [11-16] have studied the equivalence between different iterative schemes: Şolutz $[13,14]$ proved that Picard, Mann, Ishikawa, and Noor iteration schemes are equilvalent for quasi-contractive operators. Recently, Chugh and Kumar [17] proved that, for quasi-contractive operators satisfying (1.13), Picard, Mann, Ishikawa, Noor, and SP iterative schemes are equivalent.

Fixed-point iterative schemes are designed to be applied in solving equations arising in physical formulation but there is no systematic study of numerical aspects of these iterative schemes. In computational mathematics, it is of vital interest to know which of the given iterative scheme converges faster to a desired solution, commonly known as rate of convergence. Rhoades in [18] compared the Mann and Ishikawa iterative schemes by concerning their rate of convergences. He illustrated the difference in the rate of convergence for increasing and deceasing functions (see also [19]). However, Olatinwo [5] proved the stability of Kirk-Mann and Kirk-Ishikawa iterative schemes for the following operator which is more general than (1.13). Indeed, he employed the following contractive definition: there exist $a \in$ $[0,1)$ and a monotone increasing function $\varphi: R^{+} \rightarrow R^{+}$with $\varphi(0)=0$, such that

$$
\|T x-T y\| \leq \varphi(\|x-T x\|)+a\|x-y\| \quad \forall x, y \in X .
$$

Motivated by the work of Olatinow [5] and Phuengrattana and Suantai [7], in this paper, we introduce the Kirk-CR and Kirk-SP iterative schemes and study the strong convergence of these iterative schemes for quasi-contractive operators satisfying (1.14). Moreover, by using $\mathrm{C}++$ programming, comparison for rate of convergences between the above-mentioned Kirk type iterative schemes is also shown for increasing, decreasing, sublinear, superlinear, and oscillatory functions, respectively. 


\section{Main Results}

We will need the following lemmas and definition in the sequel.

Lemma 2.1 (see [10]). If $\delta$ is a real number such that $0 \leq \delta<1$ and $\left\{\epsilon_{n}\right\}_{n=0}^{\infty}$ is a sequence of nonnegative numbers such that $\lim _{n \rightarrow \infty} \in_{n}=0$, then, for any sequence of positive numbers $\left\{u_{n}\right\}_{n=0}^{\infty}$ satisfying

$$
u_{n+1} \leq \delta u_{n}+\epsilon_{n}, \quad n=0,1,2, \ldots,
$$

one has $\lim _{n \rightarrow \infty} u_{n}=0$.

Lemma 2.2 (see [5]). Let $(X,\|\cdot\|)$ be a normed linear space and let $T: X \rightarrow X$ be a selfmap of $X$ satisfying (1.13). Let $\varphi: R^{+} \rightarrow R^{+}$be a subadditive, monotone increasing function such that $\varphi(0)=0, \varphi(L u) \leq L \varphi(u), L \geq 0, u \in R^{+}$. Then, for all $i \in N, L \geq 0$ and for all $x, y \in X$,

$$
\left\|T^{i} x-T^{i} y\right\| \leq \sum_{j=1}^{i}\left(\begin{array}{l}
i \\
j
\end{array}\right) a^{i-j} \varphi^{j}(\|x-T x\|)+a^{i}\|x-y\| .
$$

Definition 2.3 (see [20]). Suppose $\left\{a_{n}\right\}$ and $\left\{b_{n}\right\}$ are two real convergent sequences with limits $a$ and $b$, respectively. Then, $\left\{a_{n}\right\}$ is said to converge faster than $\left\{b_{n}\right\}$ if

$$
\lim _{n \rightarrow \infty}\left|\frac{a_{n}-a}{b_{n}-b}\right|=0
$$

Now, we define Kirk-SP and Kirk-CR iterative schemes as follows. Let $X$ be a Banach space, $T: X \rightarrow X$ a selfmap of $X$ and $x_{0} \in X$. Then, the sequence $\left\{x_{n}\right\}_{n=0}^{\infty}$ defined by

$$
\begin{gathered}
x_{n+1}=\alpha_{n, 0} y_{n}+\sum_{i=1}^{k} \alpha_{n, i} T^{i} y_{n}, \quad \sum_{i=0}^{k} \alpha_{n, i}=1, \\
y_{n}=\beta_{n, 0} z_{n}+\sum_{r=1}^{s} \beta_{n, r} T^{r} z_{n}, \quad \sum_{j=0}^{s} \beta_{n, j}=1, \\
z_{n}=\sum_{l=0}^{t} \gamma_{n, l} T^{l} x_{n}, \quad \sum_{l=0}^{t} \gamma_{n, l}=1, \quad n=0,1,2, \ldots,
\end{gathered}
$$


is called Kirk-SP iterative scheme and the sequence $\left\{x_{n}\right\}_{n=0}^{\infty}$ defined by

$$
\begin{gathered}
x_{n+1}=\alpha_{n, 0} y_{n}+\sum_{i=1}^{k} \alpha_{n, i} T^{i} y_{n}, \quad \sum_{i=0}^{k} \alpha_{n, i}=1, \\
y_{n}=\beta_{n, 0} T x_{n}+\sum_{r=1}^{s} \beta_{n, r} T^{r} z_{n}, \quad \sum_{r=0}^{s} \beta_{n, r}=1, \\
z_{n}=\sum_{l=0}^{t} \gamma_{n, l} T^{l} x_{n}, \quad \sum_{l=0}^{t} \gamma_{n, l}=1, \quad n=0,1,2 \ldots,
\end{gathered}
$$

is called Kirk-CR iterative scheme, where $k, s$, and $t$ are fixed integers with $k \geq s \geq t$ and $\alpha_{n, i}$, $\beta_{n, r}, \gamma_{n, l}$ are sequences in $[0,1]$ satisfying $\alpha_{n, i} \geq 0, \alpha_{n, 0} \neq 0, \beta_{n, r} \geq 0, \beta_{n, 0} \neq 0, \gamma_{n, l} \geq 0, \gamma_{n, 0} \neq 0$.

Remarks 2. (5) Putting $t=s=0$ in (2.4), we obtain the Kirk-Mann iterative scheme (1.9).

(6) Putting $s=0, k=1, t=0$ in (2.4), we get usual Mann iterative scheme (1.3). With $\sum_{i=0}^{1} \alpha_{n, i}=1, \alpha_{n, 1}=\alpha_{n}$.

(7) Putting $s=0, t=0$, and $\alpha_{n, i}=\alpha_{i}$ in (2.4), we obtain the usual Kirk's iterative scheme (1.4).

(8) Putting $s=t=1$ in (2.4) and (2.5), we obtain the SP (1.10) and CR (1.11) iterative schemes, respectively.

We now prove our main results.

Theorem 2.4. Let $(X,\|\cdot\|)$ be a normed linear space and $T: X \rightarrow X$ a selfmap of $X$ satisfying the contractive condition (1.14) and $\varphi: R^{+} \rightarrow R^{+}$a subadditive monotone increasing function such that $\varphi(0)=0$ and $\varphi(L u) \leq L \varphi(u), L \geq 0, u \in R^{+}$. Let $x_{0} \in X$ and $\left\{x_{n}\right\}_{n=0}^{\infty}$ be the Kirk-SP iterative scheme defined by (2.4). Suppose that $T$ has a fixed point $p$. Then, the Kirk-SP iterative scheme converges strongly to $p$.

Proof. Using Kirk-SP iterative scheme (2.4) and Lemma 2.1, we have

$$
\begin{aligned}
\left\|x_{n+1}-p\right\| & \leq \alpha_{n, 0}\left\|y_{n}-p\right\|+\sum_{i=1}^{k} \alpha_{n, i}\left\|T^{i} y_{n}-p\right\| \\
& \leq \alpha_{n, 0}\left\|y_{n}-p\right\|+\sum_{i=1}^{k} \alpha_{n, i}\left\{\sum_{j=1}^{i}\left(\begin{array}{l}
i \\
j
\end{array}\right) a^{i-j} \varphi^{j}(\|p-T p\|)+a^{i}\left\|y_{n}-p\right\|\right\} \\
& =\alpha_{n, 0}\left\|y_{n}-p\right\|+\left(\sum_{i=1}^{k} \alpha_{n, i} a^{i}\right)\left\|y_{n}-p\right\| \\
& =\left(\sum_{i=0}^{k} \alpha_{n, i} a^{i}\right)\left\|y_{n}-p\right\| .
\end{aligned}
$$


Now, we have the following estimates:

$$
\begin{aligned}
\left\|y_{n}-p\right\| & \leq \beta_{n, 0}\left\|z_{n}-p\right\|+\sum_{r=1}^{s} \beta_{n, r}\left\|T^{r} z_{n}-p\right\| \\
& \leq \beta_{n, 0}\left\|z_{n}-p\right\|+\sum_{r=1}^{s} \beta_{n, r}\left\{\sum_{j=1}^{r}\left(\begin{array}{c}
r \\
j
\end{array}\right) a^{r-j} \varphi^{j}(\|p-T p\|)+a^{r}\left\|z_{n}-p\right\|\right\} \\
& =\left(\sum_{r=0}^{k} \beta_{n, r} a^{r}\right)\left\|z_{n}-p\right\|, \\
\left\|z_{n}-p\right\| & \leq \gamma_{n, l}\left\|x_{n}-p\right\|+\sum_{l=1}^{t} \gamma_{n, l}\left\|T^{l} x_{n}-p\right\| \\
& \leq \gamma_{n, l}\left\|x_{n}-p\right\|+\sum_{l=1}^{t} \gamma_{n, l}\left\{\sum_{j=1}^{l}\left(\begin{array}{l}
l \\
j
\end{array}\right) a^{l-j} \varphi^{j}(\|p-T p\|)+a^{l}\left\|x_{n}-p\right\|\right\} \\
& =\left(\sum_{l=0}^{t} \gamma_{n, l} a^{l}\right)\left\|x_{n}-p\right\| .
\end{aligned}
$$

It follows from (2.6), (2.7) that

$$
\left\|x_{n+1}-p\right\| \leq\left(\sum_{i=0}^{k} \alpha_{n, i} a^{i}\right)\left(\sum_{r=0}^{s} \beta_{n, i} a^{i}\right)\left(\sum_{l=0}^{t} r_{n, l} a^{l}\right)\left\|x_{n}-p\right\| .
$$

Since $a^{i} \in[0,1)$ and $\sum_{i=0}^{k} \alpha_{n, i}=\sum_{r=0}^{s} \beta_{n, r}=\sum_{l=0}^{t} \gamma_{n, l}=1$, hence

$$
\left(\sum_{i=0}^{k} \alpha_{n, i} a^{i}\right)\left(\sum_{r=0}^{s} \beta_{n, r} a^{r}\right)\left(\sum_{l=0}^{t} \gamma_{n, l} a^{l}\right)<\left(\sum_{i=0}^{k} \alpha_{n, i}\right)\left(\sum_{r=0}^{s} \beta_{n, r}\right)\left(\sum_{l=0}^{t} \gamma_{n, l}\right)=1 .
$$

Using (2.9) and Lemma 2.1, (2.8) yields $\lim _{n \rightarrow \infty} x_{n}=p$. Thus, Kirk-SP iterative scheme converges strongly to $p$.

Theorem 2.5. Let $(X,\|\cdot\|)$ be a normed linear space and $T: X \rightarrow X$ a selfmap of $X$ satisfying the contractive condition (1.14) and $\varphi: R^{+} \rightarrow R^{+}$a subadditive monotone increasing function such that $\varphi(0)=0$ and $\varphi(L u) \leq L \varphi(u), L \geq 0, u \in R^{+}$. Let $x_{0} \in X$ and $\left\{x_{n}\right\}_{n=0}^{\infty}$ be the Kirk-CR iterative scheme defined by (2.5). Suppose that $T$ has a fixed point $p$. Then, the Kirk-CR iterative scheme converges strongly to $p$.

Proof. Using Kirk-CR iterative scheme (2.5) and Lemma 2.2, we have

$$
\left\|x_{n+1}-p\right\| \leq\left(\sum_{i=0}^{k} \alpha_{n, i} a^{i}\right)\left\|y_{n}-p\right\|
$$


Now, we have the following estimates:

$$
\begin{aligned}
\left\|y_{n+1}-p\right\| & \leq \beta_{n, o}\left\|T x_{n}-p\right\|+\sum_{r=1}^{s} \beta_{n, r}\left\|T^{r} z_{n}-p\right\| \\
& \leq a \beta_{n, 0}\left\|x_{n}-p\right\|+\sum_{r=1}^{s} \beta_{n, r}\left\{\sum_{j=1}^{r}\left(\begin{array}{c}
r \\
j
\end{array}\right) a^{r-j} \varphi^{j}(\|p-T p\|)+a^{r}\left\|z_{n}-p\right\|\right\} \\
& =a \beta_{n, 0}\left\|x_{n}-p\right\|+\left(\sum_{r=1}^{s} \beta_{n, r} a^{r}\right)\left\|z_{n}-p\right\| \\
\left\|z_{n}-p\right\| & \leq\left(\sum_{l=0}^{t} \gamma_{n, l} a^{l}\right)\left\|x_{n}-p\right\| .
\end{aligned}
$$

It follows from (2.10), (2.11) that

$$
\begin{aligned}
\left\|x_{n+1}-p\right\| & \leq a\left(\sum_{i=0}^{k} \alpha_{n, i} a^{i}\right) \beta_{n, 0}\left\|x_{n}-p\right\|+\left(\sum_{i=0}^{k} \alpha_{n, i} a^{i}\right)\left(\sum_{r=1}^{s} \beta_{n, r} a^{r}\right)\left(\sum_{l=0}^{t} \gamma_{n, l} a^{l}\right)\left\|x_{n}-p\right\| \\
& =\left(\sum_{i=0}^{k} \alpha_{n, i} a^{i}\right)\left[a \beta_{n, 0}+\left(\sum_{r=1}^{s} \beta_{n, r} a^{r}\right)\left(\sum_{l=0}^{t} \gamma_{n, l} a^{l}\right)\right]\left\|x_{n}-p\right\| .
\end{aligned}
$$

Since $a^{i} \in[0,1)$ and $\sum_{i=0}^{k} \alpha_{n, i}=\sum_{r=0}^{s} \beta_{n, r}=\sum_{l=0}^{t} \gamma_{n, l}=1$ with $\alpha_{n, 0} \neq 0, \beta_{n, 0} \neq 0, \gamma_{n, 0} \neq 0$, hence

$$
\begin{aligned}
\left(\sum_{i=0}^{k} \alpha_{n, i} a^{i}\right)\left[a \beta_{n, 0}+\left(\sum_{r=1}^{s} \beta_{n, r} a^{r}\right)\left(\sum_{l=0}^{t} \gamma_{n, l} a^{l}\right)\right] & <\left(\sum_{i=0}^{k} \alpha_{n, i}\right)\left[\beta_{n, 0}+\left(\sum_{r=1}^{s} \beta_{n, r}\right)\left(\sum_{l=0}^{t} \gamma_{n, l}\right)\right] \\
& =\beta_{n, 0}+\sum_{r=1}^{s} \beta_{n, r}=1 .
\end{aligned}
$$

Using (2.13) and Lemma 2.1, (2.12) yields $\lim _{n \rightarrow \infty} x_{n}=p$. Thus, Kirk-CR iterative scheme converges strongly to $p$.

Theorem 2.6. Let $(X,\|\cdot\|)$ be a normed linear space and $T: X \rightarrow X$ a selfmap of $X$ satisfying the contractive condition (1.14) and $\varphi: R^{+} \rightarrow R^{+}$a subadditive monotone increasing function such that $\varphi(0)=0$ and $\varphi(L u) \leq L \varphi(u), L \geq 0, u \in R^{+}$. Let $x_{0} \in X$ and $\left\{x_{n}\right\}_{n=0}^{\infty}$ be the Kirk-Noor iterative scheme defined by (1.9). Suppose that $T$ has a fixed point $p$. Then, the Kirk-Noor iterative scheme converges strongly to $p$. 
Journal of Applied Mathematics

Proof. Using Kirk-Noor iterative scheme (1.9) and Lemma 2.2, we have

$$
\begin{aligned}
\left\|x_{n+1}-p\right\| & \leq \alpha_{n, 0}\left\|x_{n}-p\right\|+\sum_{i=1}^{k} \alpha_{n, i}\left\|T^{i} y_{n}-p\right\| \\
& \leq \alpha_{n, 0}\left\|x_{n}-p\right\|+\sum_{i=1}^{k} \alpha_{n, i}\left\{\sum_{j=1}^{i}\left(\begin{array}{c}
i \\
j
\end{array}\right) a^{i-j} \varphi^{j}(\|p-T p\|)+a^{i}\left\|y_{n}-p\right\|\right\} \\
& =\alpha_{n, 0}\left\|x_{n}-p\right\|+\left(\sum_{i=1}^{k} \alpha_{n, i} a^{i}\right)\left\|y_{n}-p\right\| .
\end{aligned}
$$

Now, we have the following estimates:

$$
\begin{aligned}
\left\|y_{n}-p\right\| & \leq \beta_{n, 0}\left\|x_{n}-p\right\|+\sum_{r=1}^{s} \beta_{n, r}\left\|T^{r} z_{n}-p\right\| \\
& \leq \beta_{n, 0}\left\|x_{n}-p\right\|+\sum_{r=1}^{s} \beta_{n, r}\left\{\sum_{j=1}^{r}\left(\begin{array}{l}
r \\
j
\end{array}\right) a^{r-j} \varphi^{j}(\|p-T p\|)+a^{r}\left\|z_{n}-p\right\|\right\} \\
& =\beta_{n, 0}\left\|x_{n}-p\right\|+\left(\sum_{r=1}^{k} \beta_{n, r} a^{r}\right)\left\|z_{n}-p\right\|, \\
\left\|z_{n}-p\right\| & \leq \gamma_{n, l}\left\|x_{n}-p\right\|+\sum_{l=1}^{t} \gamma_{n, l}\left\|T^{l} x_{n}-p\right\| \\
& \leq \gamma_{n, l}\left\|x_{n}-p\right\|+\sum_{l=1}^{t} \gamma_{n, l}\left\{\sum_{j=1}^{l}\left(\begin{array}{l}
l \\
j
\end{array}\right) a^{l-j} \varphi^{j}(\|p-T p\|)+a^{l}\left\|x_{n}-p\right\|\right\} \\
& =\left(\sum_{l=0}^{t} \gamma_{n, l} a^{l}\right)\left\|x_{n}-p\right\| .
\end{aligned}
$$

It follows from (2.14), (2.15) that

$$
\begin{aligned}
\left\|x_{n+1}-p\right\| & \leq \alpha_{n, 0}\left\|x_{n}-p\right\|+\left(\sum_{i=1}^{k} \alpha_{n, i} a^{i}\right) \beta_{n, 0}\left\|x_{n}-p\right\| \\
& +\left(\sum_{i=1}^{k} \alpha_{n, i} a^{i}\right)\left(\sum_{r=1}^{s} \beta_{n, i} a^{i}\right)\left(\sum_{l=0}^{t} \gamma_{n, l} a^{l}\right)\left\|x_{n}-p\right\| \\
& <\left[\alpha_{n, 0}+\left(1-\alpha_{n, 0}\right) \beta_{n, 0}+\left(1-\alpha_{n, 0}\right)\left(1-\beta_{n, 0}\right)\right]\left\|x_{n}-p\right\|=\left\|x_{n}-p\right\| .
\end{aligned}
$$

Using Lemma (2.2), (2.16) yields $\lim _{n \rightarrow \infty} x_{n}=p$. Thus, Kirk-Noor iterative scheme converges strongly to $p$. 


\section{Results on Fastness of Kirk-Type Iterative Schemes for Quasi-Contractive Operators}

In [20], Berinde showed that Picard iteration is faster than Mann iteration for quasicontractive operators satisfying (1.14). In [21], Qing and Rhoades by taking example showed that Ishikawa iteration is faster than Mann iteration for a certain quasi-contractive operator. Ciric et al. [22], by providing an example, showed that Noor iterative scheme can be faster than Mann and Ishikawa iterative schemes for some quasi-contractive operator. Recently, Hussian et al. [23], provided an example of a quasi-contractive operator for which the iterative scheme due to Agarwal et al. is faster than Mann and Ishikawa iterative schemes.

Now, by providing Example 3.1, we prove that the decreasing order of Kirk-type iterative schemes is as follows: Kirk-SP, Kirk-CR, Kirk-Noor, Kirk-Ishikawa, and Kirk-Mann iterative scheme.

However, after interchanging the parameters the decreasing order of Kirk-type iterative schemes is as follows: Kirk-CR, Kirk-SP, Kirk-Noor, Kirk-Ishikawa, and Kirk-Mann.

Example 3.1. Let $T:[0,1] \rightarrow[0,1]:=x / 2, \alpha_{n, 1}=\beta_{n, 1}=\gamma_{n, 1}=\alpha_{n, 0}=\beta_{n, 0}=\gamma_{n, 0}=4 / \sqrt{n}$, $n=1,2, \ldots, n_{0}$ for some $n_{0} \in N$ and $\alpha_{n, 2}=\beta_{n, 2}=\gamma_{n, 2}=1-8 / \sqrt{n}, n \geq n_{0}$.

It is clear that $T$ is a quasi-contractive operator satisfying (1.14) with a unique fixed point 0 . Also, it is easy to see that Example 3.1 satisfies all the conditions of Theorems 2.4, 2.5, and 2.6.

Proof. Let $n \geq 64$ and $u_{0}=x_{0}$ with $x_{0} \neq 0$. Then, for Kirk-Mann and Kirk-Ishikawa iterative schemes, we have

$$
\begin{gathered}
u_{n+1}=\prod_{i=64}^{n}\left(\frac{1}{4}+\frac{4}{\sqrt{i}}\right) u_{0}, \\
x_{n+1}=\prod_{i=64}^{n}\left(\frac{1}{16}+\frac{5}{\sqrt{i}}\right) x_{0} .
\end{gathered}
$$

Now, consider

$$
\left|\frac{x_{n+1}}{u_{n+1}}\right|=\left|\frac{\prod_{i=64}^{n}(1 / 16+5 / \sqrt{i}) x_{0}}{\prod_{i=64}^{n}(1 / 4+4 / \sqrt{i}) u_{0}}\right|=\left|\prod_{i=64}^{n}\left[1-\frac{(3 / 16-1 / \sqrt{i})}{(1 / 4+4 / \sqrt{i})}\right]\right|=\left|\prod_{i=64}^{n}\left[1-\frac{(3 \sqrt{i}-16)}{(4 \sqrt{i}+64)}\right]\right| .
$$

It is easy to see that

$$
0 \leq \lim _{n \rightarrow \infty} \prod_{i=64}^{n}\left[1-\frac{(3 \sqrt{i}-16)}{(4 \sqrt{i}+64)}\right] \leq \lim _{n \rightarrow \infty} \prod_{i=64}^{n}\left(1-\frac{1}{i}\right)=\lim _{n \rightarrow \infty} \frac{63}{n}=0 .
$$

Hence, $\lim _{n \rightarrow \infty}\left|x_{n+1} / p_{n+1}\right|=0$. 
Therefore, by Definition 2.3, Kirk-Ishikawa iterative scheme converges faster than Kirk-Mann iterative scheme to the fixed point 0 of $T$.

Similarly,

$$
\begin{aligned}
\left|\frac{x_{n+1}(\text { Kirk-Noor })}{x_{n+1}(\text { Kirk-Ishikawa })}\right| & =\left|\frac{\prod_{i=64}^{n}(1 / 64+21 / 4 \sqrt{i}) x_{0}}{\prod_{i=64}^{n}(1 / 16+5 / \sqrt{i}) x_{0}}\right| \\
& =\left|\prod_{i=64}^{n}\left[1-\frac{(3 / 64-1 / 4 \sqrt{i})}{(1 / 16+5 / \sqrt{i})}\right]\right| \\
& =\left|\prod_{i=64}^{n}\left[1-\frac{(3 \sqrt{i}-16)}{(4 \sqrt{i}+320)}\right]\right|
\end{aligned}
$$

with

$$
0 \leq \lim _{n \rightarrow \infty} \prod_{i=64}^{n}\left[1-\frac{(3 \sqrt{i}-16)}{(4 \sqrt{i}+320)}\right] \leq \lim _{n \rightarrow \infty} \prod_{i=64}^{n}\left(1-\frac{1}{i}\right)=\lim _{n \rightarrow \infty} \frac{63}{n}=0
$$

implies

$$
\lim _{n \rightarrow \infty}\left|\frac{x_{n+1}(\text { Kirk-Noor })}{x_{n+1}(\text { Kirk-Ishikawa })}\right|=0 .
$$

Therefore, by Definition 2.3, Kirk-Noor iterative scheme converges faster than Kirk-Ishikawa iterative scheme to the fixed point 0 of $T$.

Again, similarly

$$
\begin{aligned}
\left|\frac{x_{n+1}(\text { Kirk-CR })}{x_{n+1}(\text { Kirk-Noor })}\right| & =\left|\frac{\prod_{i=64}^{n}(1 / 64+1 / \sqrt{i}+12 / i) x_{0}}{\prod_{i=64}^{n}(1 / 64+21 / 4 \sqrt{i}) x_{0}}\right| \\
& =\left|\prod_{i=64}^{n}\left[1-\frac{(17 / 4 \sqrt{i}-12 / i)}{(1 / 64+21 / 4 \sqrt{i})}\right]\right| \\
& =\left|\prod_{i=64}^{n}\left[1-\frac{64(17 \sqrt{i}-48)}{(4 i+1344 \sqrt{i})}\right]\right|
\end{aligned}
$$


with

$$
0 \leq \lim _{n \rightarrow \infty} \prod_{i=64}^{n}\left[1-\frac{64(17 \sqrt{i}-48)}{(4 i+1344 \sqrt{i})}\right] \leq \lim _{n \rightarrow \infty} \prod_{i=64}^{n}\left(1-\frac{1}{i}\right)=\lim _{n \rightarrow \infty} \frac{63}{n}=0,
$$

implies

$$
\lim _{n \rightarrow \infty}\left|\frac{x_{n+1}(\text { Kirk-CR })}{x_{n+1}(\text { Kirk-Noor })}\right|=0
$$

It shows Kirk-CR iterative scheme converges faster than Kirk-Noor iterative scheme to the fixed point 0 of $T$.

Again, let $n \geq 300$. Then, for Kirk-CR iterative scheme, we have

$$
x_{n+1}=\prod_{i=300}^{n}\left(\frac{1}{64}+\frac{3}{4 \sqrt{i}}+\frac{12}{n}+\frac{64}{i^{3 / 2}}\right) x_{0} .
$$

So,

$$
\begin{aligned}
\left|\frac{x_{n+1}(\text { Kirk-SP })}{x_{n+1}(\text { Kirk-CR })}\right| & =\left|\frac{\prod_{i=300}^{n}\left(1 / 64+3 / 4 \sqrt{i}+12 / i+64 / i^{3 / 2}\right) x_{0}}{\prod_{i=300}^{n}(1 / 64+1 / \sqrt{i}+12 / i) x_{0}}\right| \\
& =\left|\prod_{i=300}^{n}\left[1-\frac{\left(1 / 4 \sqrt{i}-64 / i^{3 / 2}\right)}{1 / 64+1 / \sqrt{i}+12 / i}\right]\right| \\
& =\left|\prod_{i=300}^{n}\left[1-\frac{64 i-16384}{4 i^{3 / 2}+256 i+3072 \sqrt{i}}\right]\right|
\end{aligned}
$$

It is easy to see that

$$
0 \leq \lim _{n \rightarrow \infty} \prod_{i=300}^{n}\left[1-\frac{64 i-16384}{4 i^{3 / 2}+256 i+3072 \sqrt{i}}\right] \leq \lim _{n \rightarrow \infty} \prod_{i=300}^{n}\left[1-\frac{1}{i}\right]=\lim _{n \rightarrow \infty} \frac{299}{n}=0
$$

Hence, we have $\lim _{n \rightarrow \infty} \mid x_{n+1}\left(\right.$ Kirk-SP) $/ x_{n+1}($ Kirk-CR $) \mid=0$. It shows Kirk-SP iterative scheme converges faster than Kirk-CR iterative scheme to the fixed point 0 of $T$.

The following example shows comparison of simple iterative schemes with their corresponding Kirk-type iterative schemes.

Example 3.2. Let $T:[0,1] \rightarrow[0,1]:=x / 2, \alpha_{n, 0}=\beta_{n, 0}=\gamma_{n, 0}=\alpha_{n, 1}=\beta_{n, 1}=\gamma_{n, 1}=4 / \sqrt{n}$, $n=1,2, \ldots, n_{0}$, for some $n_{0} \in N$ and $\alpha_{n, 3}=\beta_{n, 3}=\gamma_{n, 3}=1-8 / \sqrt{n}, n \geq n_{0}$. It is clear that $T$ is a quasi-contractive operator satisfying (1.14) with a unique fixed point 0 . Also, it is easy to see 
that Example 3.2 satisfies all the conditions of Theorems 2.4, 2.5, and 2.6. We will show the following:

(1) Kirk-Mann iterative scheme is faster than Mann iterative scheme,

(2) Kirk-Ishikawa iterative scheme is faster than Ishikawa iterative scheme,

(3) Kirk-Noor iterative scheme is faster than Noor iterative scheme,

(4) Kirk-SP iterative scheme is faster than SP iterative scheme,

(5) Kirk-CR iterative scheme is faster than CR iterative scheme.

Proof. Let $n \geq 70$ and $u_{0}=x_{0}$ with $x_{0} \neq 0$. Then, for Kirk-Mann and Mann iterative schemes, we have

$$
\begin{aligned}
& u_{n+1}=\prod_{i=64}^{n}\left(\frac{1}{4}+\frac{4}{\sqrt{n}}\right) u_{0}, \\
& x_{n+1}=\prod_{i=70}^{n}\left(1-\frac{2}{\sqrt{i}}\right) x_{0} .
\end{aligned}
$$

Now, consider

$$
\left|\frac{x_{n+1}}{u_{n+1}}\right|=\left|\frac{\prod_{i=70}^{n}(1 / 4+4 / \sqrt{i}) x_{0}}{\prod_{i=70}^{n}(1-2 / \sqrt{i}) u_{0}}\right|=\left|\prod_{i=70}^{n}\left[1-\frac{(3 / 4-6 / \sqrt{i})}{(1-2 / \sqrt{i})}\right]\right|=\left|\prod_{i=70}^{n}\left[1-\frac{(3 \sqrt{i}-24)}{(4 \sqrt{i}-8)}\right]\right| .
$$

It is easy to see that

$$
0 \leq \lim _{n \rightarrow \infty} \prod_{i=70}^{n}\left[1-\frac{(3 \sqrt{i}-24)}{(4 \sqrt{i}-8)}\right] \leq \lim _{n \rightarrow \infty} \prod_{i=70}^{n}\left(1-\frac{1}{i}\right)=\lim _{n \rightarrow \infty} \frac{69}{n}=0
$$

Hence, we have $\lim _{n \rightarrow \infty}\left|x_{n+1} / u_{n+1}\right|=0$.

It shows that Kirk-Mann iterative scheme converges faster than Mann iterative scheme to the fixed point 0 of $T$. Similarly,

$$
\begin{aligned}
\left|\frac{x_{n+1}(\text { Kirk-Ishikawa })}{x_{n+1}(\text { Ishikawa })}\right| & =\left|\frac{\prod_{i=70}^{n}(1 / 16+5 / \sqrt{i}) x_{0}}{\prod_{i=70}^{n}(1-2 / \sqrt{i}-4 / i) x_{0}}\right| \\
& =\left|\prod_{i=70}^{n}\left[1-\frac{(15 / 16-7 / \sqrt{i}-4 / i)}{(1-2 / \sqrt{i}-4 / i)}\right]\right| \\
& =\left|\prod_{i=70}^{n}\left[1-\frac{(15 i-112 \sqrt{i}-64)}{(16 i-32 \sqrt{i}-64)}\right]\right|
\end{aligned}
$$


with

$$
0 \leq \lim _{n \rightarrow \infty} \prod_{i=70}^{n}\left[1-\frac{(15 i-112 \sqrt{i}-64)}{(16 i-32 \sqrt{i}-64)}\right] \leq \lim _{n \rightarrow \infty} \prod_{i=70}^{n}\left(1-\frac{1}{i}\right)=\lim _{n \rightarrow \infty} \frac{69}{n}=0,
$$

implies

$$
\lim _{n \rightarrow \infty}\left|\frac{x_{n+1}(\text { Kirk-Ishikawa })}{x_{n+1}(\text { Ishikawa })}\right|=0
$$

It shows that Kirk-Ishikawa iterative scheme converges faster than Ishikawa iterative scheme to the fixed point 0 of $T$.

Again, similarly,

$$
\begin{aligned}
\left|\frac{\left.x_{n+1} \text { (Kirk-Noor }\right)}{x_{n+1}(\text { Noor })}\right| & =\frac{\prod_{i=70}^{n}(1 / 64+21 / 4 \sqrt{i}) x_{0}}{\prod_{i=70}^{n}\left(1-2 / \sqrt{i}-4 / i-8 / i^{3 / 2}\right) x_{0}} \\
& =\prod_{i=70}^{n}\left[1-\frac{\left(63 / 64-29 / 4 \sqrt{i}-4 / i-8 / i^{3 / 2}\right)}{1-2 / \sqrt{i}-4 / i-8 / i^{3 / 2}}\right] \\
& =\prod_{i=70}^{n}\left[1-\frac{\left(63 i^{3 / 2}-464 i-256 \sqrt{i}-512\right)}{64 i^{3 / 2}-128 i-256 \sqrt{i}-512}\right],
\end{aligned}
$$

with

$$
0 \leq \prod_{i=70}^{n}\left[1-\frac{\left(63 i^{3 / 2}-464 i-256 \sqrt{i}-512\right)}{64 i^{3 / 2}-128 i-256 \sqrt{i}-512}\right] \leq \lim _{n \rightarrow \infty} \prod_{i=70}^{n}\left[1-\frac{1}{i}\right]=\lim _{n \rightarrow \infty} \frac{69}{n}=0
$$

implies

$$
\lim _{n \rightarrow \infty}\left|\frac{x_{n+1}(\text { Kirk-Noor })}{x_{n+1}(\text { Noor })}\right|=0
$$

It shows that Kirk-Noor iterative scheme converges faster than Noor iterative scheme to the fixed point 0 of $T$. 
Journal of Applied Mathematics

Again,

$$
\begin{aligned}
\left|\frac{x_{n+1}(\text { Kirk-SP })}{x_{n+1}(\mathrm{SP})}\right| & =\left|\frac{\prod_{i=70}^{n}\left(1 / 64+3 / 4 \sqrt{i}+12 / i+64 / i^{3 / 2}\right) x_{0}}{\prod_{i=70}^{n}\left(1-6 / \sqrt{i}+12 / i-8 / i^{3 / 2}\right) x_{0}}\right| \\
& =\left|\prod_{i=70}^{n}\left[1-\frac{\left(63 / 64-27 / 4 \sqrt{i}-72 / i^{3 / 2}\right)}{\left(1-6 / \sqrt{i}+12 / i-8 / i^{3 / 2}\right)}\right]\right| \\
& =\left|\prod_{i=70}^{n}\left[1-\frac{\left(63 i^{3 / 2}-432 i-4608\right)}{64 i^{3 / 2}-384 i-768 \sqrt{i}-512}\right]\right|,
\end{aligned}
$$

with

$$
0 \leq \lim _{n \rightarrow \infty} \prod_{i=70}^{n}\left[1-\frac{\left(63 i^{3 / 2}-432 i-4608\right)}{64 i^{3 / 2}-384 i-768 \sqrt{i}-512}\right] \leq \lim _{n \rightarrow \infty} \prod_{i=70}^{n}\left(1-\frac{1}{i}\right)=\lim _{n \rightarrow \infty} \frac{69}{n}=0,
$$

implies

$$
\lim _{n \rightarrow \infty}\left|\frac{x_{n+1}(\text { Kirk-SP })}{x_{n+1}(\mathrm{SP})}\right|=0
$$

It shows that Kirk-SP iterative scheme converges faster than SP iterative scheme to the fixed point 0 of $T$.

Again,

$$
\begin{aligned}
\left|\frac{x_{n+1}(\text { Kirk-CR })}{x_{n+1}(\mathrm{CR})}\right| & =\frac{\prod_{i=70}^{n}(1 / 64+1 / \sqrt{i}+12 / i) x_{0}}{\prod_{i=70}^{n}\left(1 / 2-1 / \sqrt{i}-4 / i+8 / i^{3 / 2}\right) x} \\
& =\prod_{i=70}^{n}\left[1-\frac{\left(31 / 64-2 / \sqrt{i}-16 / i+8 / i^{3 / 2}\right)}{1 / 2-1 / \sqrt{i}-4 / i+8 / i^{3 / 2}}\right] \\
& =\prod_{i=70}^{n}\left[1-\frac{\left(31 i^{3 / 2}-128 i-1024 \sqrt{i}+512\right)}{32 i^{3 / 2}-64 i-256 \sqrt{i}+512}\right],
\end{aligned}
$$

with

$$
0 \leq \lim _{n \rightarrow \infty} \prod_{i=70}^{n}\left[1-\frac{\left(31 i^{3 / 2}-128 i-1024 \sqrt{i}+512\right)}{32 i^{3 / 2}-64 i-256 \sqrt{i}+512}\right] \leq \lim _{n \rightarrow \infty} \prod_{i=70}^{n}\left[1-\frac{1}{i}\right]=\lim _{n \rightarrow \infty} \frac{69}{n}=0
$$


implies

$$
\lim _{n \rightarrow \infty}\left|\frac{x_{n+1}(\text { Kirk-CR })}{x_{n+1}(\mathrm{CR})}\right|=0
$$

It shows that Kirk-CR iterative scheme converges faster than $\mathrm{CR}$ iterative scheme to the fixed point 0 of $T$.

\section{Applications}

In this section, with the help of computer programs in $\mathrm{C}++$, we compare the rate of convergence of Kirk-type iterative schemes, through examples. The outcome is listed in the form of Tables 1, 2, 3, 4, and 5, by taking $\alpha_{n, 1}=\beta_{n, 1}=\gamma_{n, 1}=\alpha_{n, 2}=\beta_{n, 2}=\gamma_{n, 2}=1 /(1+n)^{1 / 2}$, $\alpha_{n, 0}=1-\alpha_{n, 1}-\alpha_{n, 2}$, and $\beta_{n, 0}=1-\beta_{n, 1}-\beta_{n, 2}, \gamma_{n, 0}=1-\gamma_{n, 1}-\gamma_{n, 2}$ for all iterative schemes.

\subsection{Decreasing Cum Sublinear Functions}

The function $f:[0,1] \rightarrow[0,1]$ defined by $f(x)=\left(1-x^{3}\right)^{1 / 2}$ is a decreasing and sublinear function. By taking initial approximation $x_{0}=0.8$, the comparison of convergence of the above-mentioned iterative schemes to the exact fixed point $p=0.754878$ is listed in Table 1 .

\subsection{Increasing Functions}

Let $f:[0,2] \rightarrow[0,2]$ be defined by $f(x)=\sqrt{\left(\pi+x_{n} \sqrt{4-x_{n}^{2}}-\left(4-2 x_{n}{ }^{2}\right) \sin ^{-1}\left(x_{n} / 2\right)\right) / \pi}$. Then, $f$ is an increasing function. By taking initial approximation $x_{0}=1$, the comparison of convergence of the above-mentioned iterative schemes to the exact fixed point $p=1.15863$ of $f$ is listed in Table 2 .

\subsection{Functions with Multiple Zeros}

The function defined by $f(x)=(1-x)^{2}$ is a function with multiple zeros. By taking initial approximation $x_{0}=0.9$, the comparison of convergence of the above-mentioned iterative schemes to the exact fixed point $p=0.381966$ is listed in Table 3 .

\subsection{Superlinear Functions with Multiple Roots}

The function defined by $f(x)=2 x^{3}-7 x^{2}+8 x-2$ is a superlinear function with multiple real roots. By taking initial approximation $x_{0}=0.9$, the comparison of convergence of the above-mentioned iterative schemes to the exact fixed point $p=1$ is listed in Table 4 .

For detailed study, these programs are again executed after changing the parameters and some observations are made as given below. 
Table 1: Decreasing cum sublinear functions.

\begin{tabular}{ccccccccccc}
\hline & Kirk-CR iteration & \multicolumn{3}{c}{ Kirk-SP iteration } & \multicolumn{6}{c}{ Kirk-Noor iteration Kirk-Mann iteration Kirk-Ishikawa iteration } \\
$n$ & $f x_{n}$ & $x_{n+1}$ & $f x_{n}$ & $x_{n+1}$ & $f x_{n}$ & $x_{n+1}$ & $f x_{n}$ & $x_{n+1}$ & $f x_{n}$ & $x_{n+1}$ \\
\hline 0 & 0.69857 & 0.710807 & 0.69857 & 0.723726 & 0.69857 & 0.710384 & 0.69857 & 0.710418 & 0.69857 & 0.698227 \\
1 & 0.800542 & 0.75984 & 0.78799 & 0.75565 & 0.800941 & 0.770859 & 0.800909 & 0.765142 & 0.812157 & 0.779333 \\
2 & 0.7492 & 0.754819 & 0.754002 & 0.754877 & 0.736164 & 0.752595 & 0.743004 & 0.75401 & 0.725717 & 0.750854 \\
3 & 0.754944 & 0.754878 & $\mathbf{0 . 7 5 4 8 7 8}$ & $\mathbf{0 . 7 5 4 8 7 8}$ & 0.75745 & 0.754879 & 0.755859 & 0.754812 & 0.759396 & 0.754853 \\
4 & $\mathbf{0 . 7 5 4 8 7 8}$ & $\mathbf{0 . 7 5 4 8 7 8}$ & $\mathbf{0 . 7 5 4 8 7 8}$ & $\mathbf{0 . 7 5 4 8 7 8}$ & 0.754877 & 0.754878 & 0.754952 & 0.754866 & 0.754905 & 0.754875 \\
5 & $\mathbf{0 . 7 5 4 8 7 8}$ & $\mathbf{0 . 7 5 4 8 7 8}$ & $\mathbf{0 . 7 5 4 8 7 8}$ & $\mathbf{0 . 7 5 4 8 7 8}$ & $\mathbf{0 . 7 5 4 8 7 8}$ & $\mathbf{0 . 7 5 4 8 7 8}$ & 0.754891 & 0.754875 & 0.754881 & 0.754877 \\
6 & $\mathbf{0 . 7 5 4 8 7 8}$ & $\mathbf{0 . 7 5 4 8 7 8}$ & $\mathbf{0 . 7 5 4 8 7 8}$ & $\mathbf{0 . 7 5 4 8 7 8}$ & $\mathbf{0 . 7 5 4 8 7 8}$ & $\mathbf{0 . 7 5 4 8 7 8}$ & 0.754881 & 0.754877 & $\mathbf{0 . 7 5 4 8 7 8}$ & $\mathbf{0 . 7 5 4 8 7 8}$ \\
7 & $\mathbf{0 . 7 5 4 8 7 8}$ & $\mathbf{0 . 7 5 4 8 7 8}$ & $\mathbf{0 . 7 5 4 8 7 8}$ & $\mathbf{0 . 7 5 4 8 7 8}$ & $\mathbf{0 . 7 5 4 8 7 8}$ & $\mathbf{0 . 7 5 4 8 7 8}$ & 0.754879 & 0.754877 & $\mathbf{0 . 7 5 4 8 7 8}$ & $\mathbf{0 . 7 5 4 8 7 8}$ \\
8 & $\mathbf{0 . 7 5 4 8 7 8}$ & $\mathbf{0 . 7 5 4 8 7 8}$ & $\mathbf{0 . 7 5 4 8 7 8}$ & $\mathbf{0 . 7 5 4 8 7 8}$ & $\mathbf{0 . 7 5 4 8 7 8}$ & $\mathbf{0 . 7 5 4 8 7 8}$ & $\mathbf{0 . 7 5 4 8 7 8}$ & $\mathbf{0 . 7 5 4 8 7 8}$ & $\mathbf{0 . 7 5 4 8 7 8}$ & $\mathbf{0 . 7 5 4 8 7 8}$ \\
9 & $\mathbf{0 . 7 5 4 8 7 8}$ & $\mathbf{0 . 7 5 4 8 7 8}$ & $\mathbf{0 . 7 5 4 8 7 8}$ & $\mathbf{0 . 7 5 4 8 7 8}$ & $\mathbf{0 . 7 5 4 8 7 8}$ & $\mathbf{0 . 7 5 4 8 7 8}$ & $\mathbf{0 . 7 5 4 8 7 8}$ & $\mathbf{0 . 7 5 4 8 7 8}$ & $\mathbf{0 . 7 5 4 8 7 8}$ & $\mathbf{0 . 7 5 4 8 7 8}$ \\
10 & $\mathbf{0 . 7 5 4 8 7 8}$ & $\mathbf{0 . 7 5 4 8 7 8}$ & $\mathbf{0 . 7 5 4 8 7 8}$ & $\mathbf{0 . 7 5 4 8 7 8}$ & $\mathbf{0 . 7 5 4 8 7 8}$ & $\mathbf{0 . 7 5 4 8 7 8}$ & $\mathbf{0 . 7 5 4 8 7 8}$ & $\mathbf{0 . 7 5 4 8 7 8}$ & $\mathbf{0 . 7 5 4 8 7 8}$ & $\mathbf{0 . 7 5 4 8 7 8}$ \\
\hline
\end{tabular}

Table 2: Increasing functions.

\begin{tabular}{|c|c|c|c|c|c|c|c|c|c|c|}
\hline \multicolumn{3}{|c|}{ Kirk-CR iteration } & \multicolumn{2}{|c|}{ Kirk-SP iteration } & \multicolumn{2}{|c|}{ Kirk-Noor iteration } & \multicolumn{2}{|c|}{ Kirk-Mann iteration } & \multicolumn{2}{|c|}{ Kirk-Ishikawa iteration } \\
\hline$n$ & $f x_{n}$ & $x_{n+1}$ & $f x_{n}$ & $x_{n+1}$ & $f x_{n}$ & $x_{n+1}$ & $f x_{n}$ & $x_{n+1}$ & $f x_{n}$ & $x_{n+1}$ \\
\hline 0 & 1.103589 & 1.116858 & 1.103589 & 1.17464 & 1.103589 & 1.451792 & 1.103589 & 1.241473 & 1.103589 & 1.365883 \\
\hline 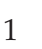 & 1.142723 & 1.158453 & 1.165002 & 1.15863 & 1.300429 & 0.997618 & 1.193272 & 1.158697 & 1.253254 & 1.076407 \\
\hline 2 & 1.158561 & 1.158631 & 1.15863 & 1.15863 & 1.102871 & 1.189602 & 1.158657 & 1.158641 & 1.128293 & 1.167741 \\
\hline 3 & 1.158631 & 1.15863 & 1.15863 & 1.15863 & 1.171096 & 1.15929 & 1.158635 & 1.158633 & 1.162237 & 1.159321 \\
\hline \pm & 1.15863 & 1.15863 & 1.15863 & 1.15863 & 1.15889 & 1.158731 & 1.158632 & 1.158631 & 1.158902 & 1.158763 \\
\hline 5 & 1.15863 & 1.15863 & 1.15863 & 1.15863 & 1.15867 & 1.158655 & 1.158631 & 1.158631 & 1.158682 & 1.158667 \\
\hline U & 1.15863 & 1.15863 & 1.15863 & 1.15863 & 1.15864 & 1.158638 & 1.158631 & 1.158631 & 1.158645 & 1.158643 \\
\hline 7 & 1.15863 & 1.15863 & 1.15863 & 1.15863 & 1.158633 & 1.158633 & 1.158631 & 1.158631 & 1.158635 & 1.158635 \\
\hline 8 & 1.15863 & 1.15863 & 1.15863 & 1.15863 & 1.158632 & 1.158632 & 1.15863 & 1.15863 & 1.158632 & 1.158632 \\
\hline 9 & 1.15863 & 1.15863 & 1.15863 & 1.15863 & 1.158631 & 1.158631 & 1.15863 & 1.15863 & 1.158631 & 1.158631 \\
\hline 10 & 1.15863 & 1.15863 & 1.15863 & 1.15863 & 1.158631 & 1.158631 & 1.15863 & 1.15863 & 1.158631 & 1.158631 \\
\hline 11 & 1.15863 & 1.15863 & 1.15863 & 1.15863 & 1.158631 & 1.158631 & 1.15863 & 1.15863 & 1.158631 & 1.158631 \\
\hline 12 & 1.15863 & 1.15863 & 1.15863 & 1.15863 & 1.15863 & 1.15863 & 1.15863 & 1.15863 & 1.158631 & 1.158631 \\
\hline 13 & 1.15863 & 1.15863 & 1.15863 & 1.15863 & 1.15863 & 1.15863 & 1.15863 & 1.15863 & 1.15863 & 1.158631 \\
\hline 14 & 1.15863 & 1.15863 & 1.15863 & 1.15863 & 1.15863 & 1.15863 & 1.15863 & 1.15863 & 1.15863 & 1.15863 \\
\hline 15 & 1.15863 & 1.15863 & 1.15863 & 1.15863 & 1.15863 & 1.15863 & 1.15863 & 1.15863 & 1.15863 & 1.15863 \\
\hline
\end{tabular}

\subsection{Oscillatory Functions}

The function defined by $f(x)=1 / x$ is an oscillatory function. By taking initial approximation $x_{0}=4$, the comparison of convergence of the above-mentioned iterative schemes to the exact fixed point $p=1$ is listed in Table 5 . 
Table 3: Functions with multiple zeros.

\begin{tabular}{ccccccccccc}
\hline \multicolumn{3}{c}{ Kirk-CR iteration } & \multicolumn{3}{c}{ Kirk-SP iteration } & \multicolumn{3}{c}{ Kirk-Noor iteration Kirk-Mann iteration Kirk-Ishikawa iteration } \\
$n$ & $f x_{n}$ & $x_{n+1}$ & $f x_{n}$ & $x_{n+1}$ & $f x_{n}$ & $x_{n+1}$ & $f x_{n}$ & $x_{n+1}$ & $f x_{n}$ & $x_{n+1}$ \\
\hline 0 & 0.01 & 0.129763 & 0.01 & 0.181407 & 0.01 & 0.194268 & 0.01 & 0.0901 & 0.01 & -0.04247 \\
1 & 0.757312 & 0.419604 & 0.670095 & 0.38479 & 0.649205 & 0.487941 & 0.827918 & 0.569045 & 1.086743 & 0.695256 \\
2 & 0.336859 & 0.382104 & 0.378484 & 0.381966 & 0.262204 & 0.363223 & 0.185722 & 0.402006 & 0.092869 & 0.341163 \\
3 & 0.381795 & 0.381966 & $\mathbf{0 . 3 8 1 9 6 6}$ & $\mathbf{0 . 3 8 1 9 6 6}$ & 0.405485 & 0.381913 & 0.357596 & 0.385139 & 0.434066 & 0.381282 \\
4 & 0.381965 & 0.381966 & $\mathbf{0 . 3 8 1 9 6 6}$ & $\mathbf{0 . 3 8 1 9 6 6}$ & 0.382031 & 0.38196 & 0.378054 & 0.382721 & 0.382812 & 0.381873 \\
5 & $\mathbf{0 . 3 8 1 9 6 6}$ & $\mathbf{0 . 3 8 1 9 6 6}$ & $\mathbf{0 . 3 8 1 9 6 6}$ & $\mathbf{0 . 3 8 1 9 6 6}$ & 0.381974 & 0.381965 & 0.381033 & 0.382195 & 0.382081 & 0.381946 \\
6 & $\mathbf{0 . 3 8 1 9 6 6}$ & $\mathbf{0 . 3 8 1 9 6 6}$ & $\mathbf{0 . 3 8 1 9 6 6}$ & $\mathbf{0 . 3 8 1 9 6 6}$ & 0.381968 & 0.381966 & 0.381683 & 0.382047 & 0.381991 & 0.38196 \\
7 & $\mathbf{0 . 3 8 1 9 6 6}$ & $\mathbf{0 . 3 8 1 9 6 6}$ & $\mathbf{0 . 3 8 1 9 6 6}$ & $\mathbf{0 . 3 8 1 9 6 6}$ & $\mathbf{0 . 3 8 1 9 6 6}$ & $\mathbf{0 . 3 8 1 9 6 6}$ & 0.381866 & 0.381998 & 0.381973 & 0.381964 \\
8 & $\mathbf{0 . 3 8 1 9 6 6}$ & $\mathbf{0 . 3 8 1 9 6 6}$ & $\mathbf{0 . 3 8 1 9 6 6}$ & $\mathbf{0 . 3 8 1 9 6 6}$ & $\mathbf{0 . 3 8 1 9 6 6}$ & $\mathbf{0 . 3 8 1 9 6 6}$ & 0.381926 & 0.38198 & 0.381968 & 0.381965 \\
9 & $\mathbf{0 . 3 8 1 9 6 6}$ & $\mathbf{0 . 3 8 1 9 6 6}$ & $\mathbf{0 . 3 8 1 9 6 6}$ & $\mathbf{0 . 3 8 1 9 6 6}$ & $\mathbf{0 . 3 8 1 9 6 6}$ & $\mathbf{0 . 3 8 1 9 6 6}$ & 0.381949 & 0.381972 & 0.381967 & 0.381966 \\
10 & $\mathbf{0 . 3 8 1 9 6 6}$ & $\mathbf{0 . 3 8 1 9 6 6}$ & $\mathbf{0 . 3 8 1 9 6 6}$ & $\mathbf{0 . 3 8 1 9 6 6}$ & $\mathbf{0 . 3 8 1 9 6 6}$ & $\mathbf{0 . 3 8 1 9 6 6}$ & 0.381958 & 0.381969 & $\mathbf{0 . 3 8 1 9 6 6}$ & $\mathbf{0 . 3 8 1 9 6 6}$ \\
11 & $\mathbf{0 . 3 8 1 9 6 6}$ & $\mathbf{0 . 3 8 1 9 6 6}$ & $\mathbf{0 . 3 8 1 9 6 6}$ & $\mathbf{0 . 3 8 1 9 6 6}$ & $\mathbf{0 . 3 8 1 9 6 6}$ & $\mathbf{0 . 3 8 1 9 6 6}$ & 0.381962 & 0.381968 & $\mathbf{0 . 3 8 1 9 6 6}$ & $\mathbf{0 . 3 8 1 9 6 6}$ \\
12 & $\mathbf{0 . 3 8 1 9 6 6}$ & $\mathbf{0 . 3 8 1 9 6 6}$ & $\mathbf{0 . 3 8 1 9 6 6}$ & $\mathbf{0 . 3 8 1 9 6 6}$ & $\mathbf{0 . 3 8 1 9 6 6}$ & $\mathbf{0 . 3 8 1 9 6 6}$ & 0.381964 & 0.381967 & $\mathbf{0 . 3 8 1 9 6 6}$ & $\mathbf{0 . 3 8 1 9 6 6}$ \\
13 & $\mathbf{0 . 3 8 1 9 6 6}$ & $\mathbf{0 . 3 8 1 9 6 6}$ & $\mathbf{0 . 3 8 1 9 6 6}$ & $\mathbf{0 . 3 8 1 9 6 6}$ & $\mathbf{0 . 3 8 1 9 6 6}$ & $\mathbf{0 . 3 8 1 9 6 6}$ & 0.381965 & 0.381966 & $\mathbf{0 . 3 8 1 9 6 6}$ & $\mathbf{0 . 3 8 1 9 6 6}$ \\
14 & $\mathbf{0 . 3 8 1 9 6 6}$ & $\mathbf{0 . 3 8 1 9 6 6}$ & $\mathbf{0 . 3 8 1 9 6 6}$ & $\mathbf{0 . 3 8 1 9 6 6}$ & $\mathbf{0 . 3 8 1 9 6 6}$ & $\mathbf{0 . 3 8 1 9 6 6}$ & 0.381965 & 0.381966 & $\mathbf{0 . 3 8 1 9 6 6}$ & $\mathbf{0 . 3 8 1 9 6 6}$ \\
15 & $\mathbf{0 . 3 8 1 9 6 6}$ & $\mathbf{0 . 3 8 1 9 6 6}$ & $\mathbf{0 . 3 8 1 9 6 6}$ & $\mathbf{0 . 3 8 1 9 6 6}$ & $\mathbf{0 . 3 8 1 9 6 6}$ & $\mathbf{0 . 3 8 1 9 6 6}$ & $\mathbf{0 . 3 8 1 9 6 6}$ & $\mathbf{0 . 3 8 1 9 6 6}$ & $\mathbf{0 . 3 8 1 9 6 6}$ & $\mathbf{0 . 3 8 1 9 6 6}$ \\
\hline
\end{tabular}

Table 4: Superlinear functions.

\begin{tabular}{|c|c|c|c|c|c|c|c|c|c|c|}
\hline \multicolumn{3}{|c|}{ Kirk-CR iteration } & \multicolumn{2}{|c|}{ Kirk-SP iteration } & \multicolumn{2}{|c|}{ Kirk-Noor iteration } & \multicolumn{2}{|c|}{ Kirk-Mann iteration } & \multicolumn{2}{|c|}{ Kirk-Ishikawa iteration } \\
\hline$n$ & $f x_{n}$ & $x_{n+1}$ & $f x_{n}$ & $x_{n+1}$ & $f x_{n}$ & $x_{n+1}$ & $f x_{n}$ & $x_{n+1}$ & $f x_{n}$ & $x_{n+1}$ \\
\hline 0 & 0.988 & 0.994372 & 0.988 & 1.083583 & 0.988 & 1.092828 & 0.988 & 1.087853 & 0.988 & 1.093597 \\
\hline 1 & 0.999968 & 0.999996 & 0.994182 & 0.993672 & 0.992983 & 0.960331 & 0.993638 & 0.959083 & 0.992879 & 0.95975 \\
\hline 2 & 1 & 1 & 0.999959 & 1.000023 & 0.998302 & 1.006115 & 0.998189 & 1.005282 & 0.998249 & 1.006211 \\
\hline 3 & 1 & 1 & 1 & 1 & 0.999963 & 1 & 0.999972 & 0.999986 & 0.999962 & 1 \\
\hline 4 & 1 & 1 & 1 & 1 & 1 & 1 & 1 & 0.999999 & 1 & 1 \\
\hline 5 & 1 & 1 & 1 & 1 & 1 & 1 & 1 & 1 & 1 & 1 \\
\hline 6 & 1 & 1 & 1 & 1 & 1 & 1 & 1 & 1 & 1 & 1 \\
\hline 7 & 1 & 1 & 1 & 1 & 1 & 1 & 1 & 1 & 1 & 1 \\
\hline 8 & 1 & 1 & 1 & 1 & 1 & 1 & 1 & 1 & 1 & 1 \\
\hline 9 & 1 & 1 & 1 & 1 & 1 & 1 & 1 & 1 & 1 & 1 \\
\hline 10 & 1 & 1 & 1 & 1 & 1 & 1 & 1 & 1 & 1 & 1 \\
\hline
\end{tabular}

\section{Observations}

\subsection{Decreasing Functions}

(1) Taking initial guess $x_{o}=0.5$ (away from the fixed point), Kirk-Mann iterative scheme converges in 9 iterations, Kirk-Ishikawa scheme converges in 9 iterations, Kirk-Noor iterative scheme converges in 6 iterations, Kirk-CR and the Kirk-SP iterative schemes converge in 5 iterations.

(2) Taking $\alpha_{n}=\beta_{n}=\gamma_{n}=1 /(1+n)^{1 / 4}$ and $x_{o}=0.8$, we observe that Kirk-Mann iterative scheme converges in 10 iterations, Kirk-Ishikawa iterative scheme converges in 12 iteration, Kirk-Noor scheme converges in 12 iterations, Kirk-CR iterative scheme converges in 8 iterations, and Kirk-SP iterative scheme converges in 6 iterations. 
Table 5: Oscillatory functions.

\begin{tabular}{cccccccccccc}
\hline \multicolumn{1}{c}{ Kirk-CR iteration } & \multicolumn{1}{c}{ Kirk-SP iteration } & \multicolumn{8}{c}{ Kirk-Noor iteration Kirk-Mann iteration Kirk-Ishikawa iteration } \\
$n$ & $f x_{n}$ & $x_{n+1}$ & $f x_{n}$ & $x_{n+1}$ & $f x_{n}$ & $x_{n+1}$ & $f x_{n}$ & $x_{n+1}$ & $f x_{n}$ & $x_{n+1}$ \\
\hline 0 & 0.25 & 0.25 & 0.25 & 0.25 & 0.25 & 0.25 & 0.25 & 0.25 & 0.25 & 0.25 \\
1 & 4 & 1.294298 & 4 & 0.966905 & 4 & 1.768962 & 4 & 2.90165 & 4 & 2.191915 \\
2 & 0.772619 & 0.995245 & 1.034228 & 1.000136 & 0.565303 & 0.889831 & 0.344631 & 1.425355 & 0.456222 & 0.833093 \\
3 & 1.004778 & 1 & 0.999864 & 1 & 1.123808 & 1 & 0.70158 & 1.063467 & 1.200346 & 1.000137 \\
4 & $\mathbf{1}$ & $\mathbf{1}$ & $\mathbf{1}$ & $\mathbf{1}$ & $\mathbf{1}$ & $\mathbf{1}$ & 0.94032 & 1.008394 & 0.999863 & 1.000015 \\
5 & $\mathbf{1}$ & $\mathbf{1}$ & $\mathbf{1}$ & $\mathbf{1}$ & $\mathbf{1}$ & $\mathbf{1}$ & 0.991676 & 1.001569 & 0.999985 & 1.000003 \\
6 & $\mathbf{1}$ & $\mathbf{1}$ & $\mathbf{1}$ & $\mathbf{1}$ & $\mathbf{1}$ & $\mathbf{1}$ & 0.998434 & 1.000384 & 0.999997 & 1.000001 \\
7 & $\mathbf{1}$ & $\mathbf{1}$ & $\mathbf{1}$ & $\mathbf{1}$ & $\mathbf{1}$ & $\mathbf{1}$ & 0.999616 & 1.000112 & 0.999999 & 1 \\
8 & $\mathbf{1}$ & $\mathbf{1}$ & $\mathbf{1}$ & $\mathbf{1}$ & $\mathbf{1}$ & $\mathbf{1}$ & 0.999888 & 1.000037 & $\mathbf{1}$ & $\mathbf{1}$ \\
9 & $\mathbf{1}$ & $\mathbf{1}$ & $\mathbf{1}$ & $\mathbf{1}$ & $\mathbf{1}$ & $\mathbf{1}$ & 0.999963 & 1.000014 & $\mathbf{1}$ & $\mathbf{1}$ \\
10 & $\mathbf{1}$ & $\mathbf{1}$ & $\mathbf{1}$ & $\mathbf{1}$ & $\mathbf{1}$ & $\mathbf{1}$ & 0.999986 & 1.000005 & $\mathbf{1}$ & $\mathbf{1}$ \\
11 & $\mathbf{1}$ & $\mathbf{1}$ & $\mathbf{1}$ & $\mathbf{1}$ & $\mathbf{1}$ & $\mathbf{1}$ & 0.999995 & 1.000002 & $\mathbf{1}$ & $\mathbf{1}$ \\
12 & $\mathbf{1}$ & $\mathbf{1}$ & $\mathbf{1}$ & $\mathbf{1}$ & $\mathbf{1}$ & $\mathbf{1}$ & 0.999998 & 1.000001 & $\mathbf{1}$ & $\mathbf{1}$ \\
13 & $\mathbf{1}$ & $\mathbf{1}$ & $\mathbf{1}$ & $\mathbf{1}$ & $\mathbf{1}$ & $\mathbf{1}$ & 0.999999 & 1 & $\mathbf{1}$ & $\mathbf{1}$ \\
14 & $\mathbf{1}$ & $\mathbf{1}$ & $\mathbf{1}$ & $\mathbf{1}$ & $\mathbf{1}$ & $\mathbf{1}$ & $\mathbf{1}$ & $\mathbf{1}$ & $\mathbf{1}$ & $\mathbf{1}$ \\
\hline
\end{tabular}

\subsection{Increasing Functions}

(1) Taking initial guess $x_{o}=0.8$ (away from the fixed point), Kirk-Mann iterative scheme converges in 19 iterations, Kirk-Ishikawa iterative scheme converges in 16 iterations, Kirk-Noor iterative scheme converges in 15 iterations, Kirk-CR iterative scheme converges in 5 iterations, and Kirk-SP iterative scheme converges in 3 iterations.

(2) Taking $\alpha_{n}=\beta_{n}=\gamma_{n}=1 /(1+n)^{1 / 4}$ and $x_{o}=1$, we observe that Kirk-Mann iterative scheme converges in 6 iterations, Kirk-Ishikawa iterative scheme converges in 11 iterations, Kirk-Noor iterative scheme converges in 13 iterations, Kirk-CR iterative scheme converges in 5 iterations, and Kirk-SP iterative scheme converges in 4 iterations.

\subsection{Functions with Multiple Zeros}

(1) Taking initial guess $x_{o}=0.6$ (near the fixed point), Kirk-Mann iterative scheme converges in 12 iterations, Kirk-Ishikawa iterative scheme converges in 10 iterations, Kirk-Noor iterative scheme converges in 8 iterations, Kirk-CR iterative scheme converges in 5 iterations and the Kirk-SP iterative scheme converges in 4 iterations.

(2) Taking $\alpha_{n}=\beta_{n}=\gamma_{n}=1 /(1+n)^{1 / 4}$ and $x_{o}=0.9$, we observe that Kirk-Mann iterative scheme converges in 9 iterations, Kirk-Ishikawa iterative scheme converges in 13 iterations, Kirk-Noor iterative scheme converges in 13 iterations, Kirk-CR iterative scheme converges in 8 iterations, and Kirk-SP iterative scheme converges in 6 iterations.

\subsection{Superlinear Functions with Multiple Roots}

(1) Taking initial guess $x_{o}=0.6$ (away from the fixed point), Kirk-Mann, Kirk-Ishikawa and Kirk-Noor iterative schemes converge in 5 iterations while Kirk-CR and the Kirk-SP schemes converge in 4 iterations. 
(2) Taking $\alpha_{n}=\beta_{n}=\gamma_{n}=1 /(1+n)^{1 / 4}$ and $x_{o}=0.9$, we observe that Kirk-Mann, KirkIshikawa, and Kirk-Noor schemes converge in 11 iterations while Kirk-CR iterative scheme converges in 4 iterations, and Kirk-SP iterative scheme converges in 3 iterations.

\subsection{Oscillatory Functions}

(1) Taking initial guess $x_{o}=0.6$ (near the fixed point), Kirk-Mann iterative scheme converges in 9 iterations, Kirk-Ishikawa, iterative scheme converges in 6 iterations while Kirk-Noor, Kirk-CR, and Kirk-SP iterative schemes converge in 5 iterations.

(2) Taking $\alpha_{n}=\beta_{n}=\gamma_{n}=1 /(1+n)^{1 / 4}$ and $x_{o}=4$, we observe that Kirk-Mann iterative scheme converges in 12 iterations, Kirk-Ishikawa iterative scheme converges in 15 iterations, Kirk-Noor iterative scheme converges in 13 iterations, Kirk-CR iterative scheme converges in 9 iterations, and Kirk-SP iterative scheme converges in 8 iterations.

\section{Conclusions}

The speed of iterative schemes depends on $\alpha_{n}, \beta_{n}$, and $\gamma_{n}$. From Tables 1-5 and obsevations made in Section 4, we conclude the following.

\subsection{Decreasing Cum Sublinear Functions}

(1) Decreasing order of rate of convergence of Kirk-type iterative schemes is as follows:

Kirk-SP, Kirk-CR, Kirk-Noor, Kirk-Ishikawa, and Kirk-Mann.

(2) For initial guess away from the fixed point, Kirk-SP and Kirk-Ishikawa iterative schemes show an increase while Kirk-CR, Kirk-Noor, and Kirk-Mann iterative schemes show no change in the number of iterations to converge.

\subsection{Increasing Functions}

(1) Decreasing order of rate of convergence of Kirk-type iterative schemes is as follows:

Kirk-SP, Kirk-CR, Kirk-Mann, Kirk-Noor, and Kirk-Ishikawa.

(2) For initial guess away from the fixed point, the number of iterations increases in case of Kirk-Mann, Kirk-Noor, and Kirk-Ishikawa iterative schemes. However, Kirk-SP and Kirk-CR schemes show no change in the number of iterations.

\subsection{Functions with Multiple Zeros}

(1) Decreasing order of rate of convergence of Kirk-type iterative schemes is as follows:

Kirk-SP, Kirk-CR, Kirk-Noor, Kirk-Ishikwa, and Kirk-Mann.

(2) For initial guess near the fixed point, Kirk-CR, Kirk-Ishikawa, and Kirk-Mann iterative schemes show a decrease while Kirk-Noor and Kirk-SP iterative schemes show no change in the number of iterations to converge. 


\subsection{Superlinear Functions}

(1) Decreasing order of rate of convergence of Kirk-type iterative schemes is as follows:

Kirk-CR, Kirk-SP, Kirk-Noor, and Kirk-Mann, while Kirk-Noor and Kirk-Ishikawa iterative schemes show equivalence.

(2) For initial guess near the fixed point, Kirk-CR iterative scheme show an increase, while Kirk-SP, Kirk-Ishikawa, Kirk-Mann, and Kirk-Noor iterative schemes show no change in the number of iterations to converge.

\subsection{Oscillatory Functions}

(1) Decreasing order of rate of convergence of Kirk type iterative schemes is as follows:

Kirk-CR, Kirk-Ishikawa, and Kirk-Mann, while Kirk-CR, Kirk-SP, and Kirk-Noor iterative schemes show equivalence.

(2) For initial guess near the fixed point, Kirk-Mann and Kirk-Ishikawa iterative schemes show a decrease, while Kirk-CR, Kirk-SP, and Kirk-Noor iterative schemes show no change in the number of iterations to converge.

Remarks 3. (9) It is observed from experiments that, on taking $k=s=t>2$, the convergence speed of each iterative scheme decreases for all type of the above-mentioned functions. Convergence speed is the highest for $k=s=t=2$.

(10) In Section 4, we have shown comparison between Kirk-type iterative schemes for decreasing functions. However, for decreasing functions of the form $f(x)=(1-x)^{m}$, $m=7,8,9 \ldots$, Kirk-type iterative schemes may not converge.

(11) Hence, Kirk-SP and Kirk-CR iterative schemes have a good potential for further applications.

\section{Acknowledgments}

The authors would like to thank the referees for valuable suggestions on the paper and $\mathrm{N}$. Hussain gratefully acknowledges the support from the Deanship of Scientific Research (DSR) at King Abdulaziz University (KAU) during this research.

\section{References}

[1] W. R. Mann, "Mean value methods in iteration," Proceedings of the American Mathematical Society, vol. 4, pp. 506-510, 1953.

[2] W. A. Kirk, "On successive approximations for nonexpansive mappings in Banach spaces," Glasgow Mathematical Journal, vol. 12, pp. 6-9, 1971.

[3] S. Ishikawa, "Fixed points by a new iteration method," Proceedings of the American Mathematical Society, vol. 44, pp. 147-150, 1974.

[4] M. A. Noor, "New approximation schemes for general variational inequalities," Journal of Mathematical Analysis and Applications, vol. 251, no. 1, pp. 217-229, 2000.

[5] M. O. Olatinwo, "Some stability results for two hybrid fixed point iterative algorithms in normed linear space," Matematichki Vesnik, vol. 61, no. 4, pp. 247-256, 2009.

[6] R. Chugh and V. Kumar, "Stability of hybrid fixed point iterative algorithms of Kirk-Noor type in normed linear space for self and nonself operators," International Journal of Contemporary Mathematical sciences, vol. 7, no. 24, pp. 1165-1184, 2012. 
[7] W. Phuengrattana and S. Suantai, "On the rate of convergence of Mann, Ishikawa, Noor and SP-iterations for continuous functions on an arbitrary interval," Journal of Computational and Applied Mathematics, vol. 235, no. 9, pp. 3006-3014, 2011.

[8] R. Chugh, V. Kumar, and S. Kumar, "Strong Convergence of a new three step iterative scheme in Banach spaces," (communicated).

[9] T. Zamfirescu, "Fix point theorems in metric spaces," Archiv der Mathematik, vol. 23, pp. 292-298, 1972.

[10] V. Berinde, "On the convergence of the Ishikawa iteration in the class of quasi contractive operators," Acta Mathematica Universitatis Comenianae, vol. 73, no. 1, pp. 119-126, 2004.

[11] A. Rafiq, "On the convergence of the three-step iteration process in the class of quasi-contractive operators," Acta Mathematica, vol. 22, no. 3, pp. 305-309, 2006.

[12] V. Berinde, Iterative Approximation of Fixed Points, Lecture Notes in Mathematics, Springer, Berlin, Germany, 2007.

[13] S. M. Şolutz, "The equivalence of Picard, Mann and Ishikawa iterations dealing with quasi-contractive operators," Mathematical Communications, vol. 10, no. 1, pp. 81-88, 2005.

[14] S. M. Şolutz, "The equivalence between Krasnoselskij, Mann, Ishikawa, Noor and multistep iterations," Mathematical Communications, vol. 12, no. 1, pp. 53-61, 2007.

[15] B. E. Rhoades and S. M. Şolutz, "The equivalence between the convergences of Ishikawa and Mann iterations for an asymptotically nonexpansive in the intermediate sense and strongly successively pseudocontractive maps," Journal of Mathematical Analysis and Applications, vol. 289, no. 1, pp. 266$278,2004$.

[16] B. E. Rhoades and S. M. Şolutz, "The equivalence between Mann-Ishikawa iterations and multistep iteration," Nonlinear Analysis, vol. 58, no. 1-2, pp. 219-228, 2004.

[17] R. Chugh and V. Kumar, "Strong convergence of SP iterative scheme for quasi-contractive operators," International Journal of Computer Applications, vol. 31, no. 5, pp. 21-27, 2011.

[18] B. E. Rhoades, "Comments on two fixed point iteration methods," Journal of Mathematical Analysis and Applications, vol. 56, no. 3, pp. 741-750, 1976.

[19] S. L. Singh, "A new approach in numerical praxis," Progress of Mathematics, vol. 32, no. 2, pp. 75-89, 1998.

[20] V. Berinde, "Picard iteration converges faster than Mann iteration for a class of quasi-contractive operators," Fixed Point Theory and Applications, no. 2, pp. 97-105, 2004.

[21] Y. Qing and B. E. Rhoades, "Comments on the rate of convergence between Mann and Ishikawa iterations applied to Zamfirescu operators," Fixed Point Theory and Applications, vol. 2008, Article ID 387504, 3 pages, 2008.

[22] L. B. Ciric, B. S. Lee, and A. Rafiq, "Faster Noor iterations," Indian Journal of Mathematics, vol. 52, no. 3, pp. 429-436, 2010.

[23] N. Hussain, A. Rafiq, B. Damjanović, and R. Lazović, "On rate of convergence of various iterative schemes," Fixed Point Theory and Applications, vol. 45, 6 pages, 2011. 


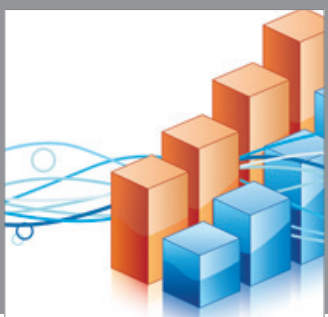

Advances in

Operations Research

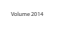

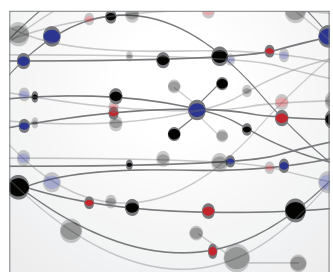

\section{The Scientific} World Journal
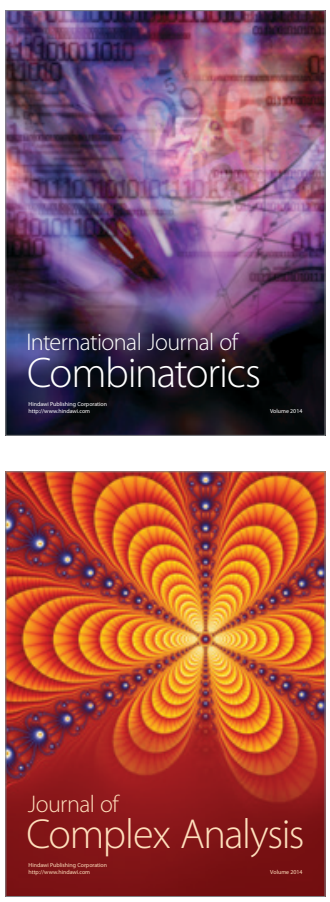

International Journal of

Mathematics and

Mathematical

Sciences
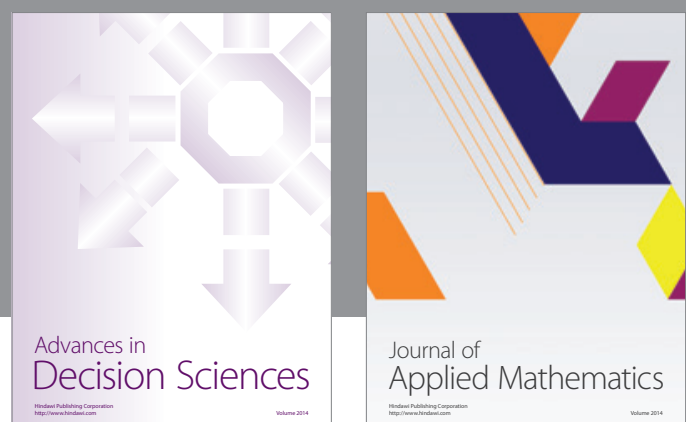

Journal of

Applied Mathematics
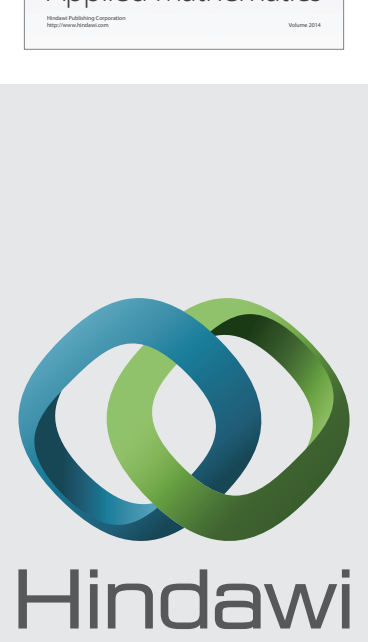

Submit your manuscripts at http://www.hindawi.com
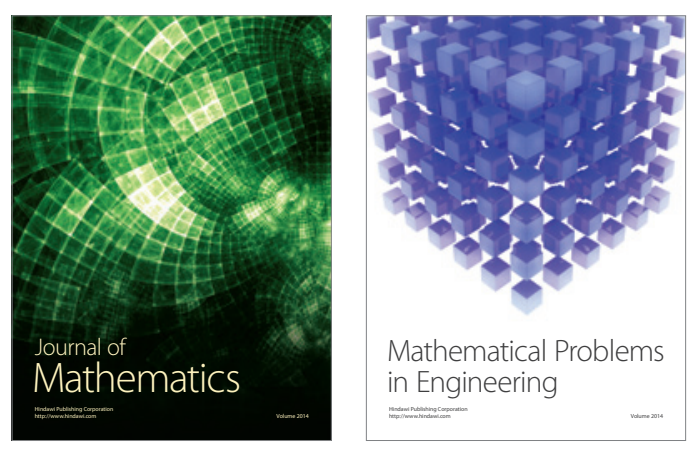

Mathematical Problems in Engineering
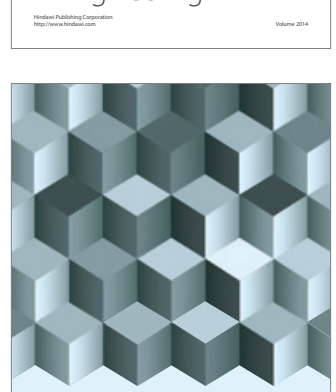

Journal of

Function Spaces
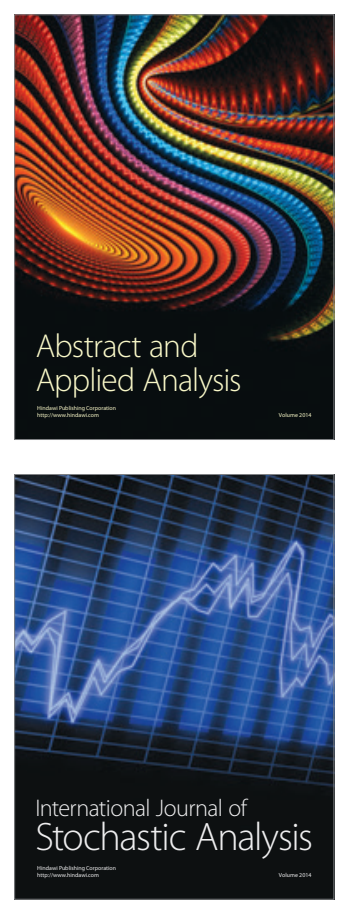

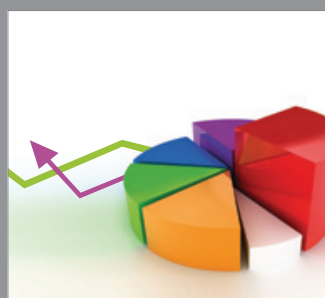

ournal of

Probability and Statistics

Promensencen
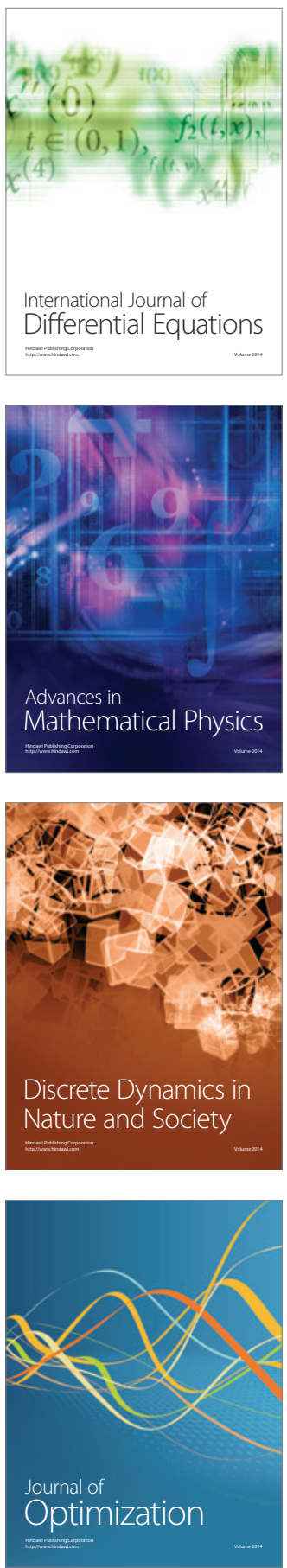